Georgian Mathematical Journal

1(1994), No. 3, 267-276

\title{
STURM'S THEOREM FOR EQUATIONS WITH DELAYED ARGUMENT
}

\author{
A.DOMOSHNITSKY
}

\begin{abstract}
Sturm's type theorems on separation of zeros of solutions are proved for the second order linear differential equations with delayed argument.
\end{abstract}

\section{INTRODUCTION}

In this article the distribution of zeros of solutions is investigated for the following differential equation with delayed arguments

$$
x^{\prime \prime}(t)+\sum_{i=1}^{m} p_{i}(t) x\left(h_{i}(t)\right)=0, \quad t \in[0,+\infty),
$$

where $p_{i}$ are locally summable nonnegative functions and $h_{i}$ are nonnegative measurable functions for $i=1, \ldots, m$.

The classical result of Sturm is the following: if $x_{1}$ and $x_{2}$ are linearly independent solutions of the ordinary differential equation

$$
x^{\prime \prime}(t)+p(t) x(t)=0, \quad t \in[0,+\infty),
$$

then between two adjacent zeros of $x_{1}$ there is one and only one zero of $x_{2}$. This article deals with the extension of the Sturm's theorem to equation (1) with delayed argument.

The first result of this type was obtained by N.V.Azbelev [1]. Namely, if for almost all $t \in[0,+\infty)$ there is at most one zero of each nontrivial solution of equation (1) on the interval $[h(t), t]$, where $h(t)=\underset{i=1, \ldots, m}{\min } h_{i}(t)$, then Sturm's theorem holds for equation (1), i.e. the interval $[h(t), t]$ must be "small enough". The generalization of this result of N.V.Azbelev to the "neutral" equation

$$
x^{\prime \prime}(t)-\sum_{j=1}^{n} q_{j}(t) x^{\prime \prime}\left(g_{j}(t)\right)+\sum_{i=1}^{m} p_{i}(t) x\left(h_{i}(t)\right)=0, \quad t \in[0,+\infty),
$$


was obtained in [3]. Our approach assumes that $\left[h_{1}(t), h_{m}(t)\right]$ is "small enough" for almost all $t \in\left[0,+\infty\right.$ ) (note that we consider the case $h_{1}(t) \leq$ $\cdots \leq h_{m}(t) \leq t$ in this article). Namely, if a solution has zero on $\left[h_{1}(t), h_{m}(t)\right]$, then its derivative has no zero on this interval.

Note the close result of S.M.Labovsky [10] for equation (1) in the case $m=1$ and another version of Sturm's separation theorem proposed by Yu.I.Domshlak $[4,5]$.

It is known [1] that the space of solutions of equation (1) is two-dimensional, the Wronskian

$$
W(t)=\left|\begin{array}{cc}
u(t) & v(t) \\
u^{\prime}(t) & v^{\prime}(t)
\end{array}\right|
$$

of a fundamental system $u, v$ of the solution (1) can vanish, zeros of $W(t)$ do not depend on a fundamental system, $W(0)$ is not equal to zero. Nonvanishing of Wronskian selects the class of homogeneous equations such that each of them is equivalent to a corresponding ordinary differential equation. In this case each nontrivial solution of equation (1) can have only finite number of zeros on any finite interval, moreover, all zeros are simple. It is also known [1] that nonvanishing of the Wronskian is equivalent to the validity of Sturm's theorem about separation of zeros.

The important part of this article concerns with estimates of the distance between adjacent zeros. These results are usually connected with Sturm's comparison theorem. Note in this connection the following investigations $[1,4-9,11,12]$.

We reduce the question about lower bounds for the distance between adjacent zeros and between zero of a solution and zero of its derivative to an estimation of the spectral radius of the corresponding completely continuous operator in the space of continuous functions, i.e. to the well-known problem of functional analysis.

Our interest in the lower bounds of this distance is connected with the problem of existence and uniqueness of a solution of boundary value problems. For example, if $b-a$ is less than the distance between adjacent zeros of solutions of (1), then the boundary value problem

$$
\begin{gathered}
x^{\prime \prime}(t)+\sum_{i=1}^{m} p_{i}(t) x\left(h_{i}(t)\right)=f(t), \quad t \in[0,+\infty), \\
x(a)=A, \quad x(b)=B,
\end{gathered}
$$

has for each $A, B, f(t)$ the unique solution.

\section{Main Results}

Let $\lambda_{\nu \mu}$ be the smallest positive characteristic number of the operator $F_{\nu \mu}: C_{[\nu, \mu]} \rightarrow C_{[\nu, \mu]}\left(C_{[\nu, \mu]}\right.$ is the space of continuous functions $x:[\nu, \mu] \rightarrow$ 
$R$ ) which is defined by

$$
\left(F_{\nu \mu} x\right)(t)=-\int_{\nu}^{\mu} G_{\nu \mu}(t, s) \sum_{i=1}^{m} p_{i}(s) x\left(h_{i}(s)\right) \gamma\left(\nu, h_{i}(s)\right) d s
$$

where

$$
\begin{aligned}
& \gamma\left(\nu, h_{i}(s)\right)=0 \text { if } h_{i}(s)<\nu, \gamma\left(\nu, h_{i}(s)\right)=1 \text { if } h_{i}(s) \geq \nu, \\
& G_{\nu \mu}(t, s)= \begin{cases}-(\mu-t)(s-\nu) /(\mu-\nu) & \text { for } \quad \nu \leq s \leq t \leq \mu, \\
-(t-\nu)(\mu-s) /(\mu-\nu) & \text { for } \quad \nu \leq t<s \leq \mu,\end{cases}
\end{aligned}
$$

$G_{\nu \mu}(t, s)$ is the Green's function of the boundary value problem

$$
x^{\prime \prime}(t)=f(t), \quad t \in[\nu, \mu], \quad x(\nu)=0, \quad x(\mu)=0 .
$$

It is clear that the operator $F_{\nu \mu}$ is positive.

Theorem 1. Let

1) the functions $h_{i}$ be nondecreasing and the inequalities $h_{i}(t) \leq h_{i+1}(t)$ hold for $i=1, \ldots, m-1$ and almost all $t \in[0,+\infty)$;

2) the functions $p_{i+1} / p_{i}$ be nondecreasing for $i=1, \ldots, m-1$;

3) at least one of the following inequalities be fulfilled

$$
\begin{gathered}
\underset{s \in\left[h_{1}(t), h_{m}(t)\right]}{\operatorname{ess} \sup _{i=1}} \sum_{i}^{m} p_{i}(s)\left[h_{m}(t)-h_{1}(t)\right]^{2}<2, \\
{\left[h_{m}(t)-h_{1}(t)\right] \int_{h_{1}(t)}^{h_{m}(t)} \sum_{i=1}^{m} p_{i}(s) d s \leq 1,}
\end{gathered}
$$

for almost all $t \in[0,+\infty)$.

Then

a) $W(t)$ doesn't vanish for $t \in[0,+\infty)$;

b) if $\nu$ and $\mu$ are two zeros of some nontrivial solution $x$ of equation (1), then $\lambda_{\nu \mu} \leq 1$

c) there is one and only one zero of the derivative of a nontrivial solution between any two adjacent zeros of this nontrivial solution.

Examples: the condition 2) of Theorem 1 is fulfilled for the following cases:

1) if $m=2, p_{1}$ is nonincreasing and $p_{2}$ is nondecreasing;

2) if $p_{i}(t)=a_{i} f(t)$, where $a_{i}=$ const, $i=1, \ldots, m$;

3) if $p_{i}(t)=a_{i} t^{i}, i=1, \ldots, m$;

4) if $p_{i}(t)=a_{i} t+b_{i}\left(a_{i}>0, b_{i}>0\right)$, where $b_{i} / a_{i}$ are nonincreasing for $i=1, \ldots, m$.

The condition that the functions $\frac{p_{i+1}}{p_{i}}$ are nondecreasing for $i=1, \ldots, m$ is essential, as the following example shows. 
Example 1. The function

$$
x(t)= \begin{cases}1-t^{2}, & 0 \leq t \leq 2, \\ 0,01 t^{2}-4,04 t+5,04, & 2<t \leq 210, \\ 2(t-5239,5)^{2}, & 210<t\end{cases}
$$

has a multiple zero at the point $t=5239,5$. This function is the solution of the equation

$$
x^{\prime \prime}(t)+p_{1}(t) x\left(h_{1}(t)\right)+x\left(h_{2}(t)\right)=0,
$$

where

$$
\begin{gathered}
h_{1}(t)=\left\{\begin{array}{ll}
0, & 0 \leq t \leq 2, \\
0,9, & t>2,
\end{array} h_{2}(t)= \begin{cases}0, & 0 \leq t \leq 2, \\
1,1, & t>2,\end{cases} \right. \\
p_{1}(t)= \begin{cases}1, & 0 \leq t \leq 210, \\
(21+32 / 10059) / 19, & t>210 .\end{cases}
\end{gathered}
$$

It is clear that $W(5239,5)=0$.

The following fact follows from Theorem 1 .

Corollary. If $m=1$ and $h_{1}$ is nondecreasing, then the assertions a),b),c) of Theorem 1 are fulfilled.

The condition that the functions $h_{i}$ are nondecreasing is essential, as the example of N.V.Azbelev [1] shows.

Let $R_{\nu \mu}(t, s), Q_{\nu \mu}(t, s)$ be Green's functions of the boundary value problems

$$
\begin{aligned}
& x^{\prime \prime}(t)=f(t), \quad t \in[\nu, \mu], \quad x(\nu)=0, \quad x^{\prime}(\mu)=0, \\
& x^{\prime \prime}(t)=f(t), \quad t \in[\nu, \mu], \quad x^{\prime}(\nu)=0, \quad x(\mu)=0
\end{aligned}
$$

respectively. It is clear that

$$
\begin{aligned}
& R_{\nu \mu}(t, s)= \begin{cases}\nu-s & \text { if } \quad \nu \leq s \leq t \leq \mu \\
\nu-t & \text { if } \quad \nu \leq t<s \leq \mu\end{cases} \\
& Q_{\nu \mu}(t, s)= \begin{cases}t-\mu & \text { if } \quad \nu \leq s \leq t \leq \mu \\
s-\mu & \text { if } \quad \nu \leq t<s \leq \mu\end{cases}
\end{aligned}
$$


Define the operators $R_{\nu \mu}, Q_{\nu \mu}: C_{[\nu, \mu]} \rightarrow C_{[\nu, \mu]}$ by the formulas

$$
\begin{gathered}
\left(R_{\nu \mu} x\right)(t)=\int_{\nu}^{\mu} R_{\nu \mu}(t, s) \sum_{i=1}^{m} p_{i}(s) x\left(h_{i}(s)\right) \gamma\left(\nu, h_{i}(s)\right) d s \\
\left(Q_{\nu \mu} x\right)(t)=\int_{\nu}^{\mu} Q_{\nu \mu}(t, s) \sum_{i=1}^{m} p_{i}(s) x\left(g_{i}(s)\right) d s
\end{gathered}
$$

here $g_{i}(i=1, \ldots, m)$ are measurable functions such that $\nu \leq g_{i}(t) \leq \mu$. Let $r_{\nu \mu}, q_{\nu \mu}$ be the smallest positive characteristic numbers of the operators $R_{\nu \mu}, Q_{\nu \mu}$ respectively.

Theorem 2. Let the conditions 1), 2) of Theorem 1 be fulfilled, $r_{h_{1}(t) h_{m}(t)}>1$ for almost all $t \in[0,+\infty), q_{h_{1}(t) h_{m}(t)}>1$ for almost all $t \in[0,+\infty)$ and all possible functions $g_{i}$ such that $g_{i}(s) \in\left[h_{1}(t), h_{m}(t)\right]$ for $s \in\left[h_{1}(t), h_{m}(t)\right], i=1, \ldots, m$. Then the assertions $\left.\left.\left.\mathrm{a}\right), \mathrm{b}\right), \mathrm{c}\right)$ of Theorem 1 are fulfilled.

Remark. The inequalities $r_{h_{1}(t) h_{m}(t)}>1$ and $q_{h_{1}(t) h_{m}(t)}>1$ for $t \in$ $[0,+\infty)$ guarantee that a solution of equation (1), having zero on the interval $\left[h_{1}(t), h_{m}(t)\right]$ has no zero of its derivative on this interval.

\section{Proofs}

We start with some auxiliary results.

Lemma 1. Let $\alpha$ be a zero of the nontrivial solution $x$ of equation (1), $\beta$ be a zero of its derivative such that $x(t)>0$ for $t \in(\alpha, \beta), \alpha<\beta$. Then there exists a set $e \subset(\alpha, \beta)$ of positive measure such that $\sum_{i=1}^{m} p_{i}(t) x\left(h_{i}(t)\right)>$ 0 for $t \in e$.

Proof of Lemma 1. Let we have on the contrary, $\sum_{i=1}^{m} p_{i}(t) x\left(h_{i}(t)\right) \leq 0$ for almost all $t \in[\alpha, \beta]$. By the theorem of Lagrange there exists $d \in(\alpha, \beta)$ such that $x^{\prime}(d)>0 . x^{\prime}(\beta)=x^{\prime}(d)+\int_{d}^{\beta} x^{\prime \prime}(s) d s=x^{\prime}(d)-\int_{d}^{\beta} \sum_{i=1}^{m} p_{i}(s) x\left(h_{i}(s)\right) d s>$ 0 , that contrast the assumption: $x^{\prime}(\beta)=0$.

Lemma 2. Let

1) $y$ be a nondecreasing function in the interval $[a, b]$;

2) $a \leq h_{1}(t) \leq h_{2}(t) \leq \cdots \leq h_{m}(t) \leq b$ for almost all $t \in[c, d] \in[a, b]$, $h_{i}$ be nondecreasing for $i=1, \ldots, m$;

3) the functions $p_{i+1} / p_{i}$ be nondecreasing for $i=1, \ldots, m-1$.

Then from the existence of a set $e \subset\left[c_{1}, d_{1}\right] \subset[c, d)$ such that $\operatorname{mes}(e)>0$ and $\sum_{i=1}^{m} p_{i}(t) y\left(h_{i}(t)\right)>0$ for $t \in e$, it follows that $\sum_{i=1}^{m} p_{i}(t) y\left(h_{i}(t)\right)>0$ for almost all $t \in\left[d_{1}, d\right]$. 
Proof of Lemma 2. Let $k$ be a number such that $y\left(h_{i}(t)\right) \geq 0$ for $t \in e$, $i \geq k$. By the condition we have the inequality $-\sum_{i=1}^{k-1} p_{i}(t) y\left(h_{i}(t)\right)<$ $\sum_{i=k}^{m} p_{i}(t) y\left(h_{i}(t)\right)$ for $t \in e$.

For all $i=1, \ldots, m y\left(h_{i}(t)\right)$ are nondecreasing since $y$ and $h_{i}$ are nondecreasing. Using the condition 3) we obtain for $t \in e$ and $r$ such that $t+r \in\left[d_{1}, d\right]:$

$$
\begin{gathered}
-\sum_{i=1}^{k-1} p_{i}(t+r) y\left(h_{i}(t+r)\right)=-\sum_{i=1}^{k-1}\left(p_{i}(t+r) / p_{i}(t)\right) p_{i}(t) y\left(h_{i}(t+r)\right) \leq \\
\leq-\left(p_{k-1}(t+r) / p_{k-1}(t)\right) \sum_{i=1}^{k-1} p_{i}(t) y\left(h_{i}(t+r)\right)< \\
<\left(p_{k-1}(t+r) / p_{k-1}(t)\right) \sum_{i=k}^{m} p_{i}(t) y\left(h_{i}(t+r)\right) \leq \\
\leq \sum_{i=k}^{m} p_{i}(t+r) y\left(h_{i}(t+r)\right) .
\end{gathered}
$$

Lemma 3. Let $[\alpha, \beta] \subset[\nu, \mu]$. Then

1) if $\lambda_{\nu \mu}>1$, then $\lambda_{\alpha \beta}>1$;

if $\lambda_{\alpha \beta} \leq 1$, then $\lambda_{\nu \mu} \leq 1$

2) if $r_{\nu \mu}>1$, then $r_{\alpha \beta}>1$;

if $r_{\alpha \beta} \leq 1$, then $r_{\nu \mu} \leq 1$

3) if $q_{\nu \mu}>1$ for each collection of functions such that $g_{i}(t) \in[\nu, \mu]$, $t \in[\nu, \mu], i=1, \ldots, m$, then $q_{\alpha \beta}>1$ each collection of functions such that $\bar{g}_{i}(t) \in[\alpha, \beta], t \in[\alpha, \beta], i=1, \ldots, m$;

if there exists a collection of functions such that $\bar{g}_{i}(t) \in[\alpha, \beta], i=$ $1, \ldots, m$, and $q_{\alpha \beta} \leq 1$, then there exists a collection of functions $g_{i}(t) \in$ $[\nu, \mu], t \in[\nu, \mu], i=1, \ldots, m$, such that $q_{\nu \mu} \leq 1$.

Proof of Lemma 3. Assertion 1) is proved in [1] and the proof of Assertion 2 ) is analogous, therefore we prove only Assertion 3).

Let us take an arbitrary collection of functions $\bar{g}_{i}(t) \in[\alpha, \beta], t \in[\alpha, \beta]$, $i=1, \ldots, m$, and denote

$$
g_{i}(t)= \begin{cases}\bar{g}_{i}(t) & \text { for } t \in[\alpha, \beta], \\ \alpha & \text { for } t \in[\alpha, \beta] .\end{cases}
$$

By condition $q_{\nu \mu}>1$ for this collection $g_{i}, i=1, \ldots, m$, the equation $x=Q_{\nu \mu} x+1$ has a positive solution $v=\lim _{n \rightarrow \infty} x_{n}$, where $x_{0}=1, x_{n+1}=$ $Q_{\nu \mu} x_{n}+1$. Since $Q_{\nu \mu}(t, s) \geq Q_{\alpha \beta}(t, s)$ for $t, s \in[\alpha, \beta]$, then $v \geq Q_{\alpha \beta} v+1$. 
Now, by the theorem about integral inequalities [1], we have $q_{\alpha \beta}>1$ for this collection $\bar{g}_{i}, i=1, \ldots, m$. The first part of the assertion 3 ) is proved.

The second part of the assertion 2) can be deduced from the first part.

Proof of Theorem 2. Let $x$ be a nontrivial solution of the equation (1). Let us consider the case $x(0)>0, x^{\prime}(0) \geq 0$ (the case $x(0) \geq 0, x^{\prime}(0)<0$ can be considered analogously).

Denote by $\beta_{1}$ the first zero of the solution's derivative, by $\alpha_{1}$ the first zero of the solution $x\left(\beta_{1}<\alpha_{1}\right.$ by our assumption). If $\beta_{1}$ or $\alpha_{1}$ doesn't exist, then the theorem is trivial.

Let us show that there exists a collection $g_{i}(t), i=1, \ldots, m$, such that $q_{\beta_{1} \alpha_{1}} \leq 1$. Really, $x$ satisfies the following equation

$$
\begin{gathered}
x(t)=-\int_{\beta_{j}}^{\alpha_{k}} Q_{\beta_{j} \alpha_{k}}(t, s) \sum_{i=1}^{m} p_{i}(s) x\left(h_{i}(s)\right) \gamma\left(\beta_{j}, h_{i}(s)\right) d s- \\
-\int_{\beta_{j}}^{\alpha_{k}} Q_{\beta_{j} \alpha_{k}}(t, s) \sum_{i=1}^{m} p_{i}(s) x\left(h_{i}(s)\right)\left[1-\gamma\left(\beta_{j}, h_{i}(s)\right)\right] d s
\end{gathered}
$$

for $t \in\left[\beta_{1}, \alpha_{1}\right]$, where $k=j=1$ and $\gamma$ is defined by (2).

Rewrite the equality (4) in the following form

$$
x(t)=-\int_{\beta_{1}}^{\alpha_{1}} Q_{\beta_{1} \alpha_{1}}(t, s) \sum_{i=1}^{m} p_{i}(s) x\left(g_{i}(s)\right) d s,
$$

where the functions $g_{i}$ such that $g_{i}(t) \in\left[\beta_{1}, \alpha_{1}\right]$.

Existence of these functions $g_{i}, i=1, \ldots, m$, follows from the next arguments. Since $x^{\prime \prime}(t)=-\sum_{i=1}^{m} p_{i}(t) x\left(h_{i}(t)\right)<0$, then $x^{\prime}$ is not increasing. Therefore $x\left(\beta_{1}\right)=\max _{t \in\left[0, \alpha_{1}\right]} x(t)$ and the set of values of function $x$ on the interval $\left[0, \beta_{1}\right]$ is included in the set of values of the function $x$ on the interval $\left[\beta_{1}, \alpha_{1}\right]$, hence the solution $g_{i}$ of the functional equation $x\left(h_{i}(t)\right)=x\left(g_{i}(t)\right)$, $t \in\left[\beta_{1}, \alpha_{1}\right]$ exists.

It is obvious that for this collection of functions $g_{i}, i=1, \ldots, m$, we have $q_{\alpha_{1} \beta_{1}} \leq 1$.

We show that $x^{\prime}\left(\alpha_{1}\right)<0$. Indeed, by the theorem of Lagrange there exists $d \in\left(\beta_{1}, \alpha_{1}\right)$ such that $x\left(\alpha_{1}\right)-x\left(\beta_{1}\right)=x^{\prime}(d)\left(\alpha_{1}-\beta_{1}\right)$. From here $x^{\prime}(d)<0$ and $x^{\prime}\left(\alpha_{1}\right)=x^{\prime}(d)+\int_{d}^{\alpha_{1}} x^{\prime \prime}(t) d t<0$.

Let $\beta_{2}$ be the first zero of the solution $x$ after $\alpha_{1}$. By Lemma 1 there exists a set $e \in\left[\alpha_{1}, \beta_{1}\right]$ with mes $(e)>0$ such that $x^{\prime \prime}(t)=-\sum_{i=1}^{m} p_{i}(t) x\left(h_{i}(t)\right)>$ 0 for $t \in e$. From here it follows that $h_{m}(t)>\alpha_{1}$ for almost all $t \geq \beta_{2}$. Since $q_{h_{1}(t) h_{m}(t)}>1$, independently of collection of functions $g_{i}, i=1, \ldots, m$ we obtain by Lemma 3 that $h_{1}(t) \geq \beta_{1}$ for almost all $t \geq \beta_{2}$. 
Next, show that $r_{\alpha_{1} \beta_{2}} \leq 1$. Indeed, on the interval $\left[\alpha_{1}, \beta_{2}\right]$ the solution $x$ of equation (1) satisfies the following integral equation

$$
\begin{gathered}
x(t)=-\int_{\alpha_{k}}^{\beta_{j}} R_{\alpha_{k} \beta_{j}}(t, s) \sum_{i=1}^{m} p_{i}(s) x\left(h_{i}(s)\right) \gamma\left(\alpha_{k}, h_{i}(s)\right) d s- \\
-\int_{\alpha_{k}}^{\beta_{j}} R_{\alpha_{k} \beta_{j}}(t, s) \sum_{i=1}^{m} p_{i}(s) x\left(h_{i}(s)\right)\left[1-\gamma\left(\alpha_{k}, h_{i}(s)\right)\right] d s,
\end{gathered}
$$

where $k=1, j=2$.

Taking $v(t)=-x(t)$, we obtain the inequality $v(t) \leq\left(R_{\alpha_{k} \beta_{j}} v\right)(t)$ for $k=1, j=2$. By the theorem about the integral inequalities (see, for example, [1]) we obtain $r_{\alpha_{1} \beta_{2}} \leq 1$.

Denote $\alpha_{2}$ the first zero of the solution $x$ after $\beta_{2}$ (if the solution $x$ hasn't a second zero $\alpha_{2}$, then Theorem 2 is trivial).

If there exist $d \in\left(\beta_{2}, \alpha_{2}\right)$ such that $h_{1}(t) \geq \alpha_{1}$ for almost all $t \geq d$, then $x\left(h_{i}(t)\right) \leq 0$ for almost all $t \in\left[d, \alpha_{2}\right]$, hence $x^{\prime \prime}(t)>0$ for almost all $t \in\left[d, \alpha_{2}\right]$.

If $\beta_{1} \leq h_{1}(t)<\alpha_{1}$, then by Lemma 2 , with the use of the condition $r_{h_{1}(t) h_{m}(t)}>1$ and Lemma 3 , we can conclude that $x^{\prime \prime}(t)>0$ for $t \in\left[\beta_{2}, d\right]$.

By the theorem of Lagrange, there exists $c \in\left(\beta_{2}, \alpha_{2}\right)$ such that $x\left(\alpha_{2}\right)-$ $x\left(\beta_{2}\right)=x^{\prime}(c)\left(\alpha_{2}-\beta_{2}\right)$, this implies $x^{\prime}(c)>0$ and $x^{\prime}\left(\alpha_{2}\right)=x^{\prime}(c)+$ $\int_{c}^{\alpha_{2}} x^{\prime \prime}(t) d t>0$. It means that $\alpha_{2}<\beta_{3}$ (we denote $\beta_{3}$ the first zero of derivative of the solution $x$ after $\alpha_{2}$ ). Now we show that $q_{\beta_{2} \alpha_{2}} \leq 1$ for some collection $g_{i}, i=1, \ldots, m$. On the interval $\left[\beta_{2}, \alpha_{2}\right]$ the solution $x$ of equation (1) satisfies equation (4) for $j=k=2$. Rewrite this equation in the following form:

$$
\begin{gathered}
x(t)=-\int_{\beta_{j}}^{\alpha_{k}} Q_{\beta_{j} \alpha_{k}}(t, s) \sum_{i=1}^{m} p_{i}(s) x\left(g_{i}(s)\right) \gamma\left(\alpha_{k-1}, h_{i}(s)\right) d s- \\
-\int_{\beta_{j}}^{\alpha_{k}} Q_{\beta_{j} \alpha_{k}}(t, s) \sum_{i=1}^{m} p_{i}(s) x\left(h_{i}(s)\right)\left[1-\gamma\left(\alpha_{k-1}, h_{i}(s)\right)\right] d s
\end{gathered}
$$

where $k=j=2, g_{i}(t) \in\left[\beta_{2}, \alpha_{2}\right]$ such that $\gamma\left(\alpha_{1}, h_{i}(t)\right)=x\left(g_{i}(t)\right)$ for $t \in\left[\beta_{2}, \alpha_{2}\right]$. The collection $g_{i}, i=1, \ldots, m$ exists since $x^{\prime \prime}(t) \geq 0$ for $t \in\left[\beta_{2}, \alpha_{2}\right]$. For, since $x^{\prime}$ isn't decreasing on the interval $\left[\beta_{2}, \alpha_{2}\right]$, therefore $\left|x\left(\beta_{2}\right)\right|=\max _{\alpha_{1} \leq t \leq \alpha_{2}}|x(t)|$ and the set of values of the function $x$ on the interval $\left[\alpha_{1}, \beta_{2}\right]$ is included in the set of values of $x$ on the interval $\left[\beta_{2}, \alpha_{2}\right]$.

It follows from [1] that $q_{\beta_{2} \alpha_{2}} \leq 1$.

The inequality $\lambda_{\alpha_{1} \alpha_{2}} \leq 1$ is proved analogously to $r_{\alpha_{1} \beta_{2}} \leq 1$.

If $\alpha_{m}$ is the last zero of the nontrivial solution, then repeating the arguments for $j, k=3,4,5, \ldots, m$, we obtain the proof of the theorem. If the solution $x$ has an infinite number of zeros, then the sequence $\alpha_{k}$ of zeros 
is unbounded. Indeed, we have proved that $\lambda_{\alpha_{k} \alpha_{k+1}} \leq 1$, this implies that [1] $\left(\alpha_{k+1}-\alpha_{k}\right) \int_{\alpha_{k}}^{\alpha_{k+1}} \sum_{i=1}^{m} p_{i}(t) d t>4$ and, consequently, the increasing sequence $\alpha_{k}$ cannot be bounded from above.

It is clear that all zeros of the solution $x$ belong to this sequence $\alpha_{k}$. In this case the repetition of our arguments completes the proof of the assertions a) and b) of Theorem 2.

The assertion c) follows from the following argument. For each $j$ we have proved that $\operatorname{sign} x(t)=\operatorname{sign} \sum_{i=1}^{m} p_{i}(t) x\left(h_{i}(t)\right)$ for $t \in\left(\beta_{j}, \alpha_{j}\right)$, this implies $x^{\prime}(t)=x^{\prime}\left(\beta_{j}\right)-\int_{\beta_{j}}^{t} \sum_{i=1}^{m} p_{i}(s) x\left(h_{i}(s)\right) d s \neq 0$ for $t \in\left(\beta_{j}, \alpha_{j}\right)$.

Proof of Theorem 1 . Theorem 1 can be obtained as a corollary of Theorem 2 .

Indeed, from the theorem about the integral inequalities [1] we have the following. If there exists a continuous positive function $v$ such that $\left.v(t)>Q_{\nu \mu} v\right)(t)\left(v(t)>\left(R_{\nu \mu} v\right)(t)\right)$ for $t \in(\nu, \mu)$, then $q_{\nu \mu}>1\left(r_{\nu \mu}>\right.$ 1). Substituting $v=1$, we obtain that the condition (4) guarantees the inequalities $r_{h_{1}(t) h_{m}(t)}>1, q_{h_{1}(t) h_{m}(t)}>1$.

If there exists a positive function $v$ such that

$$
\begin{gathered}
\varphi(t)=v^{\prime \prime}(t)+\sum_{i=1}^{m} p_{i}(t) v\left(h_{i}(t)\right) \gamma\left(v, h_{i}(t)\right) \leq 0, \quad \int_{\nu}^{\mu} \varphi(t) d t<0, \\
v^{\prime}(\nu)=0, \quad v(\mu)=0 \quad\left(v(\nu)=0, \quad v^{\prime}(\mu)=0\right)
\end{gathered}
$$

then $q_{\nu \mu}>1\left(r_{\nu \mu}>1\right)$ [2]. Substituting $v(t)=(t-2 \nu+\mu)(\mu-t)(v(t)=$ $(t-\nu)(2 \mu-\nu-t))$, we conclude that inequality (3) implies the inequality $q_{h_{1}(t) h_{m}(t)}>1\left(r_{h_{1}(t) h_{m}(t)}>1\right)$.

\section{AcKnowledgement.}

The research was supported in part by grant from the Ministry of Science and the "MA-AGARA"-special project for absorption of new immigrants, in the Department of Mathematics, Technion.

\section{REFERENCES}

1. N.V.Azbelev, About distribution of zeros of the second order differential equation with delayed argument. (Russian) Differentsial'nye Uravneniya 7(1971), No. 7, 1147-1157; English translation: Differential Equations $7(1971)$

2. N.V.Azbelev and A.I.Domoshnitsky, A question concerning linear differential inequalities, II. (Russian) Differentsial'nye Uravneniya 27(1991), No. 6, 923-931; English translation: Differential Equations 27(1991), 641647. 
3. A.I.Domoshnitsky, Extension of Sturm's theorem to apply to an equation with time-lag. (Russian) Differentsial'nye Uravneniya 19(1983), No. 9, 1475-1482; English translation: Differential Equations 19(1983), 1099-1105.

4. Yu.I.Domshlak, Sturmian comparison method in investigation of behavior of solutions for differential-operator equations. (Russian) "Elm", Baku, 1986.

5. Yu.I.Domshlak, Sturmian comparison theorem for first and second order differential equations with mixed delay of argument. (Russian) Ukrain. Mat. Zh. 34(1982), No. 2, 158-163; English translation: Ukrainian Math. J. 34(1982).

6. M.S.Du and Man Kam Kwong, Sturm comparison theorems for second order delay equations. J. Math. Anal. Appl. 152(1990), No. 2, 305-323.

7. E.A.Grove, M.R.S.Kulenovic and G.Ladas, A Myshkis-type comparison result for neutral equations. Math. Nachr. 146(1990), 195-206.

8. Man Kam Kwong and W.T.Patula, Comparison theorems of first order linear delay equations. J.Differential equations 70(1987), 275-292.

9. Yu.V.Komlenko, Sufficient conditions of regularity of the periodical boundary value problem for Hill's equations with delayed argument. (Russian) Mathematical Physics (Russian), v. 22, 5-12, "Naukova Dumka", Kiev, 1977.

10. S.M.Labovsky, About properties of fundamental system of the second order equation with delayed argument. (Russian) Trudy Tambovskogo Instituta Khimicheskogo Mashinostroeniya 6(1971), 49-52.

11. A.D.Myshkis, Linear differential equations with delayed argument. (Russian) "Nauka", Moscow, 1972.

12. D.V.Paatashvili, On oscillation of solutions of second order differential equations with delayed arguments. (Russian) Some problems of ordinary differential equations theory (Russian), Proceedings of I.N. Vekua Institute of Applied Mathematics, v. 31, 118-129. Tbilisi University Press, Tbilisi, 1988.

(Received 04.01.1993)

Author's address:

Technion-Israel Institute of Technology

Department of Mathematics

Technion City, 32000 Haifa

Israel 